\title{
Constitutive Mitochondrial DNA Copy Number in Peripheral Blood of Melanoma Families with and without CDKN2A Mutations
}

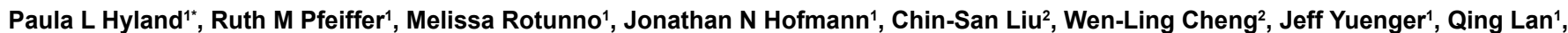 \\ Margaret A Tucker ${ }^{1}$, Alisa M Goldstein ${ }^{1}$ and Xiaohong R Yang ${ }^{1}$
}

${ }^{1}$ Division of Cancer Epidemiology and Genetics, National Cancer Institute, National Institutes of Health, Bethesda, USA

${ }^{2}$ Vascular and Genomic Research Center, Changhua Christian Hospital, Changhua 500, Taiwan

\begin{abstract}
Quantitative changes in mitochondrial DNA (mtDNA) have been associated with the risk of a number of human cancers; however, the relationship between constitutive mtDNA copy number in blood and the risk of familial cutaneous malignant melanoma (CMM) has not been reported. We measured mtDNA copy number using quantitative PCR in blood-derived DNA from $136 \mathrm{CMM}$ cases and 302 controls in 53 melanoma-prone families (23 segregating CDKN2A germline mutations). MtDNA copy number did not vary by age, sex, pigmentation characteristics, or CMM status. However, germline CDKN2A mutation carriers had significantly higher mean mtDNA copy number compared to noncarriers, particularly among CMM cases (geometric mean mtDNA copy number of 144 and 111 for carrier versus noncarrier, respectively; $\mathrm{P}=0.02$ ). When adjusting for age, sex, and familial correlation, having increasing mtDNA copy number was significantly associated with $C D K N 2 A$ mutation status among CMM cases $(\mathrm{OR}=1.47, P$ trend $=0.024)$. In particular, individuals with specific CDKN2A mutations with the potential to inactivate or reduce the level of the p16-INK4 reactive oxygen species (ROS) protective function had significantly increased mtDNA copy number levels $(P=0.035)$. Future research in prospective studies is required to validate these findings and to further investigate mtDNA copy number in both blood and melanoma tissues in relation to CMM risk and CDKN2A mutation status.
\end{abstract}

Keywords: Familial melanoma; CDKN2A; mtDNA copy number; Peripheral blood

\section{Introduction}

Cutaneous malignant melanoma (CMM) is a potentially fatal form of skin cancer with a heterogeneous etiology [1]. Established risk factors include presence and number of benign and dysplastic nevi (DN), pigmentation phenotype, and sun exposure [2]. Approximately $10 \%$ of CMM cases occur in a familial setting [1,2]. The cyclin-dependent kinase inhibitor $2 A(C D K N 2 A)$ gene is one of the two major high-risk melanoma susceptibility genes identified to date. Germline mutations of the CDKN2A gene have been described in approximately $20-40 \%$ of familial melanoma kind reds [3]. Although germline CDKN2A mutations are associated with a high risk of CMM, the penetrance of this gene is incomplete and varies by age and geographic location [4]. Further, phenotypic manifestations such as age at diagnosis, DN, number of melanomas, and co-segregation of pancreatic cancer vary significantly among mutation carriers even within a single family $[1,3]$. These findings suggest that other factors may modify the effect of CDKN2A.

Mitochondria play an essential role in numerous biological processes such as adenosine triphosphate (ATP) production via oxidative phosphorylation, iron and calcium homeostasis, and apoptosis signaling. Mitochondrial DNA (mtDNA) is an essential multicopy genome consisting of $16.6-\mathrm{kb}$ circular double-stranded DNA molecules that lacks introns, but is compacted into protein-DNA clusters called nucleoids. Due to its lack of histone protection, limited repair capacity, and close proximity to the electron transport chain, which constantly generates superoxide radicals, mtDNA is susceptible to oxidative DNA damage and exhibits high mutation rates $[5,6]$. MtDNA copy number (i.e. the total number of mtDNA genomes) per cell may increase to compensate for mtDNA damage/dysfunction and may be a marker of exposure to reactive oxygen species (ROS) and / or oxidative stress [7-9]. Alternatively, a decrease in mtDNA copy number levels could lead to decreased ATP generation by oxidative phosphorylation and concomitant enhanced glycolysis [10,11], wellknown characteristics of cancer cells.

In addition, mtDNA copy number may reflect gene-environment interactions between hereditary factors and exposures such as UV, which can affect the levels of oxidative stress [12,13]. Recently, a number of epidemiologic studies have reported that mtDNA copy number alterations in peripheral blood are associated with the risk of several cancers $[5,12,14-23]$. MtDNA copy number in blood may be relevant to familial melanoma because impaired DNA repair [24] and UV-mediated oxidative stress play a significant role in melanoma etiology [25]. Also, increased mtDNA copy number has been observed in sun-exposed skin samples compared to less exposed samples, suggesting mtDNA copy number may be a potential biomarker of UV exposure [13]. Expression of p16-INK4 from CDKN2A is also induced by exogenous UV-irradiation and endogenous $\mathrm{H} 2 \mathrm{O} 2$-oxidative stress in murine cells, and p16-INK4 can suppress ROS in vitro via a mechanism independent of its cell cycle function [25]. Loss of the p16INK4 protective ROS function in vitro has also been associated with particular familial melanoma-associated point mutations in CDKN2A [26].

*Corresponding author: Paula L. Hyland, Genetic Epidemiology Branch, Division of Cancer Epidemiology and Genetics, National Institutes of Health, Bethesda, Maryland 20892, USA, Tel: 240-276-7225; E-mail: hylandpl@mail.nih.gov

Received May 05, 2014; Accepted Jun 21, 2014; Published Jun 26, 2014

Citation: Hyland PL, Pfeiffer RM, Rotunno M, Hofmann JN, Liu CS, et al. (2014) Constitutive Mitochondrial DNA Copy Number in Peripheral Blood of Melanoma Families with and without CDKN2A Mutations. J Carcinogene Mutagene S4:006 doi:10.4172/2157-2518.S4-006

Copyright: (c) 2014 Hyland PL, et al. This is an open-access article distributed under the terms of the Creative Commons Attribution License, which permits unrestricted use, distribution, and reproduction in any medium, provided the original author and source are credited. 
Therefore, studying mtDNA copy number in whole blood and its relationship with melanoma risk and $C D K N 2 A$ status may improve our understanding of melanoma etiology in these families, To our knowledge, no study has investigated the relationship between mtDNA copy number and the risk of familial CMM nor the influence of CDKN2A germline mutations on constitutive mtDNA copy number in peripheral blood. In this study, we determined the relative mtDNA copy number from whole blood from 53 melanoma-prone families $(30$ CDKN2A-mutation negative [CDKN2A-] and 23 CDKN2A-mutation positive $[C D K N 2 A+])$ and evaluated whether constitutive mtDNA copy number varied by CMM and germline CDKN2A mutation status.

\section{Materials and Methods}

\section{Study population}

The details of this family study have previously been described $[27,28]$. In brief, U.S. families with at least two living first degree relatives with a history of invasive melanoma were ascertained through health care professionals or self-referrals. All participants in the study underwent a full-body skin examination to characterize phenotypes and completed risk factor questionnaires for sun-related exposures such as tanning ability. All diagnoses of melanoma were confirmed by histologic review of pathologic material and pathology reports. CMM cases and controls with and without CDKN2A mutations were selected from families based on the availability of blood DNA. All study participants were Caucasians, and the study population was comprised of 139 CMM cases (61 CDKN2A+ and $78 C D K N 2 A-)$ and 310 controls (45CDKN2A+ and $265 C D K N 2 A-$ ). The study was approved by the National Cancer Institute Clinical Center Institutional Review Board and conducted according to the Declaration of Helsinki. Informed consent was obtained from all participants.

\section{Quantitation of relative mtDNA copy number}

For this investigation we used DNA samples previously extracted from whole blood using a standardized protocol for total genomic DNA. All extracted DNA samples were quantitated using a NanoDrop (Thermo Scientific, Wilmington, DE, USA). We used a quantitative real-time PCR assay, as described previously in detail [29], to measure relative mtDNA copy number. All laboratory personnel performing the assays were blinded to the case-control status of all DNA samples. Briefly, a fluorescence-based quantitative PCR assay was used to determine the threshold cycle number of a mitochondrial gene (ND1) and a nuclear gene $(\beta$-globin gene, $H B B)$. The ratio of $N D 1$ and $H B B$ threshold cycle numbers, estimated using a linear regression model, is proportional to the mtDNA copy number in each cell. All samples were run in triplicate and for quality control purposes, 38 blinded duplicate samples were interspersed among the test samples. The overall coefficient of variation (CV) for mtDNA copy number for duplicate replicas was $7 \%$ and the intra-class correlation coefficient was 0.99 . The median overall CV for all test samples was $3 \%$. We were unable to assay samples from three cases and eight controls, therefore analyses were carried out on 302 controls and 136 cases.

\section{Categorization of $C D K N 2 A$ germline mutations}

p16-INK4 can suppress ROSs in vitro via a mechanism independent of its cell cycle function [25]. Loss of this ROS protective function has also been associated with particular familial melanoma-associated point mutations in $C D K N 2 A$ in vitro [26]. Thus specific CDKN2A mutations with the potential to inactivate or reduce the level of the p16-INK4 ROS protective function may result in increased constitutive
mtDNA copy number levels in the blood of CDKN2A+ mutation carriers compared to non-carriers. Jenkins et al. [26] reported that $\mathrm{R} 87 \mathrm{~W}$ (and/or mutation at amino acid R87) impairs both the cell cycle and ROS protective functions of p16-INK4 in vitro. In contrast, V126D alters only cell cycle function. In addition, independent data suggests that the cell cycle function of p16-INK4 is dependent on amino acids 84-103 within the third ankyrin motif of the protein [30], and melanoma-associated mutations that selectively impair oxidative regulation may be located outside of this region [23]. To evaluate if potential ROS-related CDKN2A mutations might influence mtDNA copy number in blood, we compared the relative mean mtDNA copy number between $C D K N 2 A$-carrier controls $(\mathrm{n}=30)$ from 13 CDKN2A+ families that carried mutations located at amino acid R87 and outside the 84-103 amino acid sequence to all non-carrier controls in our study $(n=262)$. Individuals with mutations $(n=10)$ that affected p14ARF primary structure only, located within amino acids 84-103 (G101W), or were previously suggested to alter only cell cycle function and not ROS protection (V126D) were not included in this group. We also compared the relative mean mtDNA copy number between $C D K N 2 A$ carrier cases $(\mathrm{n}=36)$ from $14 C D K N 2 A+$ families that carried mutations located at amino acid R87 and outside the 84-103 amino acid sequence to all non-carrier cases in our study $(\mathrm{n}=77)$ (Table 1$)$.

\section{Statistical analyses}

Characteristics of cases and controls were compared using logistic regression models. The relative mean mtDNA copy number levels were natural log-transformed to more closely follow a normal distribution; results of analyses of mtDNA copy number as a continuous variable are reported as geometric means. In addition, we also used box-cox power transformed data $(\lambda=-0.75)$ to further test our results in terms of significant findings. Linear regression was used to assess whether the distribution of relative mean mtDNA copy number differed significantly between spouse controls and unaffected family members and between cases and controls. We further examined mtDNA copy number as a continuous variable among controls using a multivariable linear regression to assess how age at blood draw, CDKN2A mutation status and other CMM risk factors influenced mtDNA copy number levels. Normality of residuals was assessed using the Cramer von Mises test statistic. To assess our main hypothesis, we estimated odds ratios (ORs) and 95\% confidence intervals (95\% CIs) for the association between mtDNA copy number and CMM status using unconditional logistic regression adjusting for age at blood draw $(<35,35-45,45-$ 50 and $>50$ years), sex and CDKN2A mutation status. Subjects were categorized into quartiles based on the distribution of mtDNA copy number among controls. For all models, we accounted for familial correlations among family members in the variance computation and P-values using a generalized estimating equation (GEE) with the independence working correlation matrix [31]. Tests for trend were computed assuming linear trend across different quartiles. Associations were also evaluated separately for individuals with $(+)$ and without (-) CDKN2A germline mutations. To assess how specific germline CDKN2A mutations might influence constitutive mtDNA copy number, we collated individuals with specific CDKN2A mutations into a single group (Table 1) and compared mtDNA copy number among carrier controls to all non-carrier controls, and among carrier cases to non-carrier cases from $C D K N 2 A+$ families using the GEE regression model adjusting for age at blood draw, sex, and familial correlation in the variance. All tests were two-sided. Statistical analyses were carried out using SAS software version 9.1 (SAS Institute, Cary, NC). 


\section{Results}

In total, we analyzed $136 \mathrm{CMM}$ cases $(59 \mathrm{CDKN} 2 A+$ and 77 $C D K N 2 A-)$ and 302 controls or unaffected individuals (40 CDKN2A+ and $262 C D K N 2 A-)$ from 53 melanoma-prone families. As expected, CDKN2A carrier status, DN, number of moles, number of freckles, solar injury, skin type and MC1R genotype were all significantly associated with the risk of CMM in these families (Table 2).

Overall, the mean mtDNA copy number did not differ significantly between non-carrier spouse controls $(\mathrm{n}=104)$ and related family controls that are CDKN2A- $(\mathrm{n}=158)(\mathrm{P}=0.72)$, and we therefore combined these two groups as non-carrier controls. After the adjustment for familial correlation, mtDNA copy number did not show significant associations with age at blood draw, sex, or any CMM risk factor in non-carrier controls (Supplementary Table 1).

In general, mtDNA copy number levels were lower among cases compared to controls across most categories including age at blood draw and sex, but the difference was not significant when comparing all CMM cases to all controls $(\mathrm{P}=0.094)$ (Supplementary Table 2). Although the highest quartile of mtDNA copy number levels (Q4, $>164$ ) showed a suggestive association with reduced risk of CMM in the multivariate model $(\mathrm{OR}=0.57,95 \% \mathrm{CI}=0.31-1.07, \mathrm{P}=0.084)$, the association did not follow the trend (Ptrend $=0.169$, Supplementary Table 3). In contrast, we observed that mean mtDNA copy number levels were significantly higher in all cases with $C D K N 2 A$ germline mutations ( $\mathrm{n}=59$, mean copy number=144) compared to mutationnegative cases $(n=77$, mean copy number $=111)(P=0.01$, Table 3$)$. Increasing mtDNA copy number levels was associated with a significant higher risk of being a $C D K N 2 A+$ carrier case $(\mathrm{OR}=1.48$, Ptrend $=0.024$, Table 4). A similar pattern was observed when we compared mean mtDNA copy number in CMM cases carrying potential ROS-related mutations $(\mathrm{n}=36)$ to non-carrier cases $(\mathrm{n}=77)(\mathrm{P}=0.035)$ (Table 3). Similarly, CMM-unaffected individuals carrying potential ROS-related mutations $(n=30)$ also had higher mtDNA copy number compared to non-carrier controls $(n=262)$, although the difference was not statistically significant after adjustment for age at blood draw, sex and familial correlation $(\mathrm{P}=0.16$, Table 3$)$.

\section{Discussion}

In this study, we evaluated whether mtDNA copy number in blood-derived DNA varied by CMM and germline CDKN2A mutation status in melanoma-prone families. Our results suggest that melanoma cases carrying CDKN2A mutations, including those with ROS-related mutations, had significantly higher mtDNA copy number levels compared to non-carrier cases. To our knowledge, this is the first study evaluating mtDNA copy number levels in blood in relation to CMM risk and germline $C D K N 2 A$ mutations.

Overall, we did not see a significant difference in mtDNA copy number between cases and controls, although the highest quartile of mtDNA copy number levels (Q4, >164) showed a suggestive association with reduced risk of CMM in our melanoma families. Both increasing and decreasing mtDNA copy number in blood have been previously associated with cancer risk [5,12,14-23]; this has been suggested to reflect the variation in sample size, study population, study design, or tumor-specificity. In our study, blood was collected after melanoma diagnosis for the vast majority of melanoma cases $(\mathrm{n}=110)$, which prohibited us from evaluating $\mathrm{mtDNA}$ copy number in pre-diagnostic samples. Nevertheless, we did observe higher mtDNA copy number levels in blood samples from CMM cases obtained before diagnosis $(n=26$; mean mtDNA copy number $=153)$ compared to postdiagnosis $(n=110$; mean mtDNA copy number $=118)$, suggesting that the observed reduced mtDNA copy number may be a melanoma-

\begin{tabular}{|c|c|c|c|}
\hline Family Code & CDKN2A Mutation & Base change & p16 AA change \\
\hline A & 23ins 24 & g.32-33ins9-32 & p.M1-S8dup \\
\hline A7 & M53I & g.159G >C / g.202G>C & p.M53I \\
\hline AP & IVS2-105A/G & g.458-105A>G & p.156-157del \\
\hline AY & D153spl & g.384_457del & p.R128fs137X \\
\hline B & L16R & g. $47 \mathrm{~T}>\mathrm{G}$ & p.L16R \\
\hline C & M53I & g.159G >C / g.202G>C & p.M53I \\
\hline D & R58X & g. $172 \mathrm{C}>\mathrm{T} / \mathrm{g} .215 \mathrm{C}>\mathrm{T}$ & p.R58X \\
\hline D1 & R87W & g. $259 \mathrm{C}>\mathrm{T} / \mathrm{g} .302 \mathrm{C}>\mathrm{T}$ & p.R87W \\
\hline$E$ & N71S & g. $212 A>G / g .255 A>G$ & p.N71S \\
\hline $\mathrm{F}$ & R87P & g.260G >C / g.303G >C & p.R87P \\
\hline N & 167del31 & g.167_197del / g.210_240del & p.G67fsX145 \\
\hline $\mathrm{O}$ & 225del19 & g.225_243del / g.268_286del & p.A76fs139X \\
\hline $\mathrm{P}$ & 240del14 & g.240_253del / g.283_296del & p.1-80p16:100-133p14 \\
\hline Q & IVS2+1 & g. $457+1 G>T$ & p.Ex1a-Ex3delEx2 / p.R128fs137X \\
\hline $\mathrm{AH}$ & Intron 1 & IVS1b+2T>C & NONE* $^{*}$ \\
\hline AN & Exon $1 \mathrm{~b}$ del & Exon1b del & NONE* \\
\hline $\mathrm{G}, \mathrm{CC}, \mathrm{H}$ and I & G101W & g.301G>T / g.344G>T & p.G101W \\
\hline $\mathrm{J}, \mathrm{K}$ and $\mathrm{L}$ & V126D & g.377T $>A$ & p.V126D \\
\hline
\end{tabular}

Table 1: Germline CDKN2A mutations in melanoma-prone families. CMM unaffected and affected individuals with germline $C D K N 2 A$ mutations from mutation-positive melanoma families were collated into a single group (shaded gray) with potential to influence ROS protection and cell cycle function. Mutations were grouped based on location outside the cell-cycle dependent amino acid sequence 84-103 and previous evidence suggesting a dual impact on ROS protection and cell cycle functions in vitro $[23,28]$. Families with individuals with CDKN2A mutations which only affected p14ARF primary structure, located within the amino acid sequence $84-103$ (G101W) or were previously suggested to alter only cell cycle function and not ROS protection (V126D) were not included in this group and/or analyzed separately due to insufficient numbers. p16AA changes designated $\mathrm{NONE}^{*}=$ Mutation that alters primary structure of p14ARF only. The study did not include any unaffected individuals from the mutation-positive family $Q$. 


\begin{tabular}{|c|c|c|c|c|c|}
\hline & \multicolumn{2}{|c|}{$\begin{array}{l}\text { Unaffected Individuals } \\
\qquad(n=302)\end{array}$} & \multicolumn{2}{|c|}{$\begin{array}{l}\text { CMM Cases } \\
(n=136)\end{array}$} & \multirow[t]{2}{*}{$P$} \\
\hline & $\mathrm{N}$ & $\%$ & $\mathrm{~N}$ & $\%$ & \\
\hline \multicolumn{6}{|l|}{ Age at blood draw } \\
\hline$<35$ & 74 & 24.5 & 31 & 22.8 & \\
\hline $35-45$ & 89 & 29.5 & 33 & 24.3 & \\
\hline $46-50$ & 35 & 11.6 & 19 & 14.0 & \\
\hline $50+$ & 104 & 34.4 & 53 & 39.0 & 0.616 \\
\hline \multicolumn{6}{|l|}{ Gender } \\
\hline Female & 172 & 57.0 & 73 & 53.7 & \\
\hline Male & 130 & 43.1 & 63 & 46.3 & 0.537 \\
\hline \multicolumn{6}{|l|}{ CDKN2A } \\
\hline Non-Carrier & 262 & 86.8 & 77 & 56.6 & \\
\hline Carrier & 40 & 13.3 & 59 & 43.4 & $<0.0001$ \\
\hline \multicolumn{6}{|l|}{ Dysplastic nevi } \\
\hline Unaffected & 143 & 47.4 & 2 & 1.5 & \\
\hline Affected & 119 & 39.4 & 103 & 97.7 & $<0.0001$ \\
\hline \multicolumn{6}{|l|}{ Moles } \\
\hline $0-24$ & 93 & 30.8 & 5 & 3.6 & \\
\hline $25-99$ & 105 & 34.8 & 27 & 19.9 & \\
\hline $100+$ & 81 & 28.8 & 81 & 59.6 & $<0.0001$ \\
\hline \multicolumn{6}{|l|}{ Freckles } \\
\hline Non/few & 92 & 30.4 & 17 & 12.5 & \\
\hline Moderate & 51 & 16.9 & 19 & 14.0 & \\
\hline Many & 75 & 24.8 & 49 & 36.0 & $<0.0001$ \\
\hline \multicolumn{6}{|l|}{ Solar injury } \\
\hline None/mild & 184 & 60.9 & 52 & 38.2 & \\
\hline Moderate & 58 & 19.2 & 38 & 27.9 & \\
\hline Severe & 38 & 12.6 & 23 & 16.9 & 0.0002 \\
\hline \multicolumn{6}{|l|}{ Tanning ability } \\
\hline Tan/Little burn & 147 & 48.7 & 44 & 14.6 & \\
\hline Burn/Little tan & 118 & 39.1 & 68 & 22.5 & 0.0116 \\
\hline \multicolumn{6}{|l|}{ Skin type } \\
\hline Dark/medium & 87 & 28.8 & 17 & 12.5 & \\
\hline Pale/fair & 187 & 61.9 & 96 & 70.6 & 0.0013 \\
\hline \multicolumn{6}{|l|}{ Eye color } \\
\hline Black/brown & 69 & 22.8 & 19 & 14.0 & \\
\hline Hazel & 62 & 20.5 & 25 & 18.4 & \\
\hline Green/gray & 24 & 7.9 & 17 & 12.5 & \\
\hline Blue & 120 & 39.7 & 52 & 38.2 & 0.1626 \\
\hline \multicolumn{6}{|l|}{ Hair Color } \\
\hline Black/brown & 124 & 41.1 & 35 & 25.7 & \\
\hline Blonde brown/light brown & 74 & 24.5 & 27 & 19.9 & \\
\hline Blonde & 47 & 15.6 & 12 & 8.8 & \\
\hline Red & 30 & 9.9 & 13 & 9.6 & 0.1598 \\
\hline
\end{tabular}

Table 2: Distribution of age, gender, CDKN2A, pigmentation phenotype, and sun exposure variables in 53 melanoma-prone families by CMM status. P-values were obtained by comparing $\mathrm{CMM}$ cases to unaffected individuals using logistic regression with a generalized estimating equation and adjusting for familial correlation in the variance.

related effect rather than a marker of risk, and/or may be attributable to a general immune response to melanoma in our cases. As surgery remains the mainstay of melanoma therapy, this result is unlikely to reflect a response to treatment in cases [32].

Oxidative stress has been shown to increase mtDNA content in vitro [8]. Expression of p16-INK4 from CDKN2A is induced by exogenous UV-irradiation and endogenous oxidative stress, and p16-INK4 can suppress the generation of ROS in vitro [25]. Different murine skin cells including melanocytes have been shown to exhibit increased levels of intracellular ROS in the context of deficiency of p16INK4, which can be restored upon re-expression. The regulation of oxidative stress by p16-INK4 was shown to occur independently of Rb or its cell cycle control function in skin cells [25]. Germline CDKN2A mutations are associated with a high risk of familial CMM and the loss of the p16-INK4 protective ROS function has been associated with specific CDKN2A familial melanoma-associated point mutations [26] . Thus, we hypothesized that individuals with specific germline CDKN2A mutations conferring a potential reduction or elimination of p16-INK4 ROS protective function would characterize higher constitutive mtDNA copy number levels in blood DNA compared to individuals with no germline CDKN2A mutations. Consistent with our hypothesis, we observed a significantly higher mtDNA copy number in $C D K N 2 A+$ carrier cases compared to CDKN2A-mutation negative cases. The association was similar when we analyzed pre-diagnostic and post-diagnostic cases separately. Similarly, CDKN2A mutation carrier 


\begin{tabular}{|c|c|c|c|c|c|c|c|c|c|c|}
\hline \multirow[b]{2}{*}{$C D K N 2 A$} & \multicolumn{5}{|c|}{ Unaffected Individuals } & \multicolumn{5}{|c|}{ Cases } \\
\hline & $\mathbf{N}$ & Mean\# & $\begin{array}{c}\text { Range } \\
\text { (Min-Max) }\end{array}$ & $\mathbf{P}$ & $\mathbf{P}^{*}$ & $\mathbf{N}$ & Mean\# & $\begin{array}{c}\text { Range } \\
\text { (Min-Max) }\end{array}$ & $\mathbf{P}$ & $\mathbf{P}^{*}$ \\
\hline $\begin{array}{l}\text { All Non- } \\
\text { Carrier }\end{array}$ & 262 & 132 & $33-2644$ & & & 77 & 111 & $36-1588$ & & \\
\hline All Carrier & 40 & 162 & $52-1604$ & 0.080 & 0.20 & 59 & 144 & $45-1022$ & 0.024 & 0.01 \\
\hline $\begin{array}{l}\text { ROS-Related } \\
\text { Carrier }\end{array}$ & 30 & 174 & $52-781$ & 0.038 & 0.16 & 36 & 141 & $48-713$ & 0.035 & 0.04 \\
\hline
\end{tabular}

Table 3: Mean mtDNA copy number by $C D K N 2 A$ mutation status. P-values were obtained by comparing $C D K N 2 A$ mutation carriers and non-carriers by CMM status using linear regression analysis of natural log-transformed data adjusting for familial correlation in the variance using a generalized estimating equation approach $(P)$ with further adjustment for age at blood draw and sex $\left(\mathrm{P}^{*}\right)$. Using box-cox transformed $(\lambda=-0.75)$ data did not alter the results significantly. \# Geometric means (or exponentiation of log-transformed mtDNA copy number).

\begin{tabular}{|c|c|c|c|c|}
\hline \multicolumn{5}{|c|}{ CMM Cases } \\
\hline \multirow[t]{2}{*}{ mtDNACoN\# } & CDKN2A- Non-Carrier $(n=77)$ & $C D K N 2 A+$ Carrier $(n=59)$ & \multicolumn{2}{|c|}{ Model } \\
\hline & $\mathrm{N} \quad(\%)$ & $\mathrm{N} \quad(\%)$ & OR $(95 \% \mathrm{Cl})$ & $\mathrm{P}$ \\
\hline Q1 (<83) & $22 \quad(28.6)$ & $11(18.6)$ & Ref & \\
\hline Q2 (83-106) & $(26.0)$ & $17(28.2)$ & $1.69(0.66-4.4)$ & 0.273 \\
\hline Q3 (107-164) & $26 \quad(33.8)$ & $13(22.0)$ & $0.98(0.36-2.65)$ & 0.961 \\
\hline Q4 (>164) & $9 \quad(11.7)$ & $18(30.5)$ & $5.04(1.5-17.0)$ & 0.009 \\
\hline Ptrend & & & & 0.024 \\
\hline
\end{tabular}

Table 4: Association between mtDNA copy number in blood and risk of being a CDKN2A+ carrier case. Odds ratios and P-values were computed using an unconditional logistic regression model adjusted for age at blood draw, sex, and familial correlation using a generalized estimating equation approach. Abbreviations: mtDNACoN =mitochondrial DNA copy number; OR = odds ratio; $\mathrm{Cl}=$ confidence interval; SD = standard deviation; $\mathrm{Q} 1=$ quartile 1; Q2 = quartile 2; $\mathrm{Q} 3$ = quartile 3 and $\mathrm{Q} 4=$ quartile

4 ; and \# = exponentiation of log-transformed mtDNA copy number.

controls also had a slight increase in mtDNA copy number compared to non-carrier controls. Although these findings are biologically plausible, the clinical relevance of increased mtDNA copy number in peripheral blood of $C D K N 2 A+$ melanoma cases remains unclear. It is possible that an already high constitutive mtDNA copy number level occurring in $C D K N 2 A+$ melanoma cases may be further increased in tumor tissues to compensate for more mtDNA damage by oxidative stress and mitochondrial dysfunction. In line with this hypothesis, increased mtDNA copy number in serum has been correlated with TNM stage of lung cancer [33].

Consistent with previous findings, we found no significant association between mtDNA copy number and age at blood draw $[19,21,34]$ among unaffected individuals. Taking advantage of the detailed pigmentation phenotypes we have collected for our family members, we examined the influence of established CMM risk factors on mtDNA copy number among unaffected individuals. Melanin and UV irradiation have been shown to alter mtDNA copy number in human melanoma cell lines [35] and mtDNA copy number may be a potential biomarker of UV exposure, a major risk factor for CMM [13]. Increasing mtDNA copy number has been observed in sun exposed skin samples [13] and in normal skin from CMM patients with high-risk pigmentation phenotypes [36]. However, in the present study, we found no significant association for mtDNA copy number in blood with any of the CMM risk factors examined including solar injury, tanning ability and/or pigmentation phenotypes (skin type, eye color, hair color, moles, etc.), which might suggest that the ability of these factors to alter mtDNA copy number may be more important for cells directly exposed to UV irradiation. A marginally non-significant association for mtDNA copy number in blood with solar injury $(\mathrm{P}=$ 0.09) was observed in $C D K N 2 A+$ carrier controls. However, while this result is based on a small number of $C D K N 2 A+$ carrier controls $(\mathrm{n}=40)$ in our study, it suggests that the association of solar injury with mtDNA copy number in blood may also vary by CDKN2A mutations status and the potential of p16-INK4 to protect against UV-induced oxidative stress. Notably, melanoma in melanoma-prone families with $C D K N 2 \mathrm{~A}$ mutations is associated with chronic exposure to UV radiation [4], which may further exacerbate an already altered constitutive mtDNA copy via generation of ROS in skin.

While not a primary aim of our study, recent evidence suggests that telomere length is associated with mitochondrial biogenesis and function, as well as increased ROS levels [37] and that mtDNA copy number positively correlates with telomere length in blood from elderly women [38]. Also, TERT reverse transcriptase localizes to the mitochondria (mt) and mtTERT improves mitochondrial function and stress resistance, independently of its telomeric function [39]. Taking advantage of previously published telomere length data from our melanoma-prone families [40], we assessed the correlation between telomere length and mtDNA copy number in blood from the same set of controls $(\mathrm{n}=158)$, however, no significant correlation was found. Similarly, we did not observe any significant association between common genetic variants in TERT ( $\mathrm{n}=12$ tagging SNPs) [40] and mtDNA copy number (control $=158$ and cases $=77$ ) (data not shown).

This study is the first to examine mtDNA copy number in blood with risk of melanoma in melanoma-prone families with and without CDKN2A mutations as well as the influence of specific germline CDKN2A mutations on mtDNA copy number. Our findings further highlight that until we have a thorough understanding of the genetic and non-genetic factors that may influence mtDNA copy number levels, we need to be cautious when viewing mtDNA copy number in blood as a biomarker of cancer risk. 
Our families had well annotated sun exposure and pigmentation data, were strongly influenced by genetic factors, and were of Caucasian ethnicity, minimizing the influence of race and genetic background on mtDNA copy number. However, our study is limited by its small sample size. In addition, our families were ascertained primarily through selfor physician-referral, which might affect the generalizability of the results.

In conclusion, our data did not support a significant difference in mtDNA copy number in blood between CMM cases and controls. However, CMM cases (and to a lesser extent unaffected individuals) with germline $C D K N 2 A$ mutations had significantly higher mtDNA copy number suggesting that specific CDKN2A mutations can alter constitutive mtDNA copy number, possibly as a result of increased susceptibility to oxidative stress. Future research in prospective studies is required to validate our results and to investigate the functional significance of altered mtDNA copy number in both blood and melanoma tissues.

\section{Funding}

This research was funded by an Intramural Research Program Award of the National Institutes of Health, National Cancer Institute, Division of Cancer Epidemiology and Genetics (to PLH).

\section{Acknowledgements}

We are indebted to the participating families, whose generosity and cooperation have made our study possible. We also acknowledge Virginia Pichler Deborah Zametkin, and Mary Fraser for their contributions to this work.

\section{Conflict of Interest}

The authors state no conflict of interest.

\section{Reference}

1. Goldstein AM, Tucker MA (2001) Genetic epidemiology of cutaneous melanoma: a global perspective. Arch Dermatol 137: 1493-1496.

2. Tucker MA, Fraser MC, Goldstein AM, Struewing JP, King MA, et al. (2002) A natural history of melanomas and dysplastic nevi: an atlas of lesions in melanoma-prone families. Cancer 94: 3192-3209.

3. Goldstein AM (2004) Familial melanoma, pancreatic cancer and germline CDKN2A mutations. Hum Mutat 23: 630

4. Bishop DT, Demenais F, Goldstein AM, Bergman W, Bishop JN, et al. (2002) Geographical variation in the penetrance of $C D K N 2 A$ mutations for melanoma. J Natl Cancer Inst 94: 894-903.

5. Lan Q, Lim U, Liu CS, Weinstein SJ, Chanock S, et al. (2008) A prospective study of mitochondrial DNA copy number and risk of non-Hodgkin lymphoma. Blood 112: 4247-4249.

6. Carew JS, Huang P (2002) Mitochondrial defects in cancer. Mol Cancer 1: 9.

7. Mansouri A, Demeilliers C, Amsellem S, Pessayre D, Fromenty B (2001) Acute ethanol administration oxidatively damages and depletes mitochondria DNA in mouse liver, brain, heart, and skeletal muscles: Protective effects of antioxidants. J Pharmacol Exp Ther 298: 737-743.

8. Lee HC, Yin PH, Lu CY, Chi CW, Wei YH (2000) Increase of mitochondria and mitochondrial DNA in response to oxidative stress in human cells. Biochem $\mathrm{J}$ 348 Pt 2: 425-432.

9. Zhong Y, Hu YJ, Chen B, Peng W, Sun Y, et al. (2011) Mitochondria transcription factor $A$ overexpression and base excision repair deficiency in the inner ear of rats with D-galactose-induced aging. FEBS J 278: 2500-2510.

10. Jeng JY, Yeh TS, Lee JW, Lin SH, Fong TH, et al. (2008) Maintenance of mitochondrial DNA copy number and expression are essential for preservation of mitochondrial function and cell growth. J Cell Biochem 103: 347-357.

11. Shadel GS (2008) Expression and maintenance of mitochondrial DNA: new insights into human disease pathology. Am J Pathol 172: 1445-1456.
12. Xing J, Chen M, Wood CG, Lin J, Spitz MR, et al. (2008) Mitochondrial DNA content: its genetic heritability and association with renal cell carcinoma. J Nat Cancer Inst 100: 1104-1112.

13. Gebhard D, Mahler B, Matt K, Burger K, Bergemann J (2014) Mitochondria DNA copy number - but not a mitochondrial tandem CC to TT transition - is increased in sun-exposed skin. Exp Dermatol 23: 209-211.

14. Hosgood HD 3rd, Liu CS, Rothman N, Weinstein SJ, Bonner MR, et al. (2010) Mitochondrial DNA copy number and lung cancer risk in a prospective cohort study. Carcinogenesis 31: 847-849.

15. Liao LM, Baccarelli A, Shu XO, Gao YT, Ji BT, et al. (2011) Mitochondrial DNA copy number and risk of gastric cancer: a report from the Shanghai Women's Health Study. Cancer Epidemiol Biomarkers Prev 20: 1944-1949.

16. Lynch SM, Weinstein SJ, Virtamo J, Lan Q, Liu CS, et al. (2011) Mitochondrial DNA copy number and pancreatic cancer in the alpha-tocopherol beta-carotene cancer prevention study. Cancer Prev Res (Phila) 4: 1912-1919.

17. Thyagarajan B, Wang R, Barcelo H, Koh WP, Yuan JM (2012) Mitochondria copy number is associated with colorectal cancer risk. Cancer Epidemio Biomarkers Prev 21: 1574-1581.

18. Hofmann JN, Hosgood HD 3rd, Liu CS, Chow WH, Shuch B, et al. (2014) A nested case-control study of leukocyte mitochondrial DNA copy number and renal cell carcinoma in the Prostate, Lung, Colorectal and Ovarian Cancer Screening Trial. Carcinogenesis 35: 1028-1031.

19. Purdue MP, Hofmann JN, Colt JS, Hoxha M, Ruterbusch JJ, et al. (2012) A case-control study of peripheral blood mitochondrial DNA copy number and risk of renal cell carcinoma. PLoS One 7: e43149.

20. Xie H, Lev D, Gong Y, Wang S, Pollock RE, et al. (2013) Reduced mitochondria DNA copy number in peripheral blood leukocytes increases the risk of soft tissue sarcoma. Carcinogenesis 34: 1039-1043.

21. Xu E, Sun W, Gu J, Chow WH, Ajani JA, et al. (2013) Association of mitochondrial DNA copy number in peripheral blood leukocytes with risk of esophageal adenocarcinoma. Carcinogenesis 34: 2521-2524.

22. Zhao S, Yang Y, Liu J, Liu H, Ge N, et al. (2011) Association of mitochondria DNA content in peripheral blood leukocyte with hepatitis B virus-related hepatocellular carcinoma in a Chinese Han population. Cancer Sci 102: 15531558

23. Yu M, Zhou Y, Shi Y, Ning L, Yang Y, et al. (2007) Reduced mitochondrial DNA copy number is correlated with tumor progression and prognosis in Chinese breast cancer patients. IUBMB Life 59: 450-457.

24. Sarkar-Agrawal P, Vergilis I, Sharpless NE, DePinho RA, Rünger TM (2004) Impaired processing of DNA photoproducts and ultraviolet hypermutability with loss of p16INK4a or p19ARF. J Natl Cancer Inst 96: 1790-1793.

25. Jenkins NC, Liu T, Cassidy P, Leachman SA, Boucher KM, et al. (2011) The p16(INK4A) tumor suppressor regulates cellular oxidative stress. Oncogene 30: 265-274.

26. Jenkins NC, Jung J, Liu T, Wilde M, Holmen SL, et al. (2013) Familial melanomaassociated mutations in $\mathrm{p} 16$ uncouple its tumor-suppressor functions. J Invest Dermatol 133: 1043-1051.

27. Goldstein AM, Landi MT, Tsang S, Fraser MC, Munroe DJ, et al. (2005) Association of MC1R variants and risk of melanoma in melanoma-prone families with $C D K N 2 A$ mutations. Cancer Epidemiol Biomarkers Prev 14: 2208 2212.

28. Goldstein AM, Struewing JP, Chidambaram A, Fraser MC, Tucker MA (2000) Genotype-phenotype relationships in U.S. melanoma-prone families with CDKN2A and CDK4 mutations. J Natl Cancer Inst 92: 1006-1010.

29. Liu CS, Tsai CS, Kuo CL, Chen HW, Lii CK, et al. (2003) Oxidative stress-related alteration of the copy number of mitochondrial DNA in human leukocytes. Free Radic Res 37: 1307-1317.

30. Fahham N, Sardari S, Ostad SN, Vaziri B, Ghahremani MH (2010) C-termina domain of p16(INK4a) is adequate in inducing cell cycle arrest, growth inhibition and CDK4/6 interaction similar to the full length protein in HT-1080 fibrosarcoma cells. J Cell Biochem 111: 1598-1606.

31. Liang KY, Zeger SL (1986) Longitudinal Data-Analysis Using Generalized Linear-Models. Biometrika 73: 13-22.

32. Rutkowski P, Zdzienicki M, Nowecki Zl, Van Akkooi AC (2010) Surgery of primary melanomas. Cancers (Basel) 2: 824-841. 
Citation: Hyland PL, Pfeiffer RM, Rotunno M, Hofmann JN, Liu CS, et al. (2014) Constitutive Mitochondrial DNA Copy Number in Peripheral Blood of Melanoma Families with and without CDKN2A Mutations. J Carcinogene Mutagene S4:006 doi:10.4172/2157-2518.S4-006

Page 7 of 5

33. Hou YL, Chen JJ, Wu YF, Xue CJ, Li FZ, et al. (2013) Clinical significance of serum mitochondrial DNA in lung cancer. Clin Biochem 46: 1474-1477.

34. Yu M, Wan YF, Zou QH (2012) Cell-free circulating mitochondrial DNA in the serum: a potential non-invasive biomarker for Ewing's sarcoma. Arch Med Res 43: 389-394.

35. Swalwell H, Latimer J, Haywood RM, Birch-Machin MA (2012) Investigating the role of melanin in UVA/UVB- and hydrogen peroxide-induced cellular and mitochondrial ROS production and mitochondrial DNA damage in human melanoma cells. Free Radic Biol Med 52: 626-634.

36. Hubbard K, Steinberg ML, Hill H, Orlow I (2008) Mitochondrial DNA deletions in skin from melanoma patients. Ethn Dis 18: S2-38-43.
37. Sahin E, Colla S, Liesa M, Moslehi J, Müller FL, et al. (2011) Telomere dysfunction induces metabolic and mitochondrial compromise. Nature 470 359-365.

38. Kim JH, Kim HK, Ko JH, Bang H, Lee DC (2013) The relationship between leukocyte mitochondrial DNA copy number and telomere length in communitydwelling elderly women. PLoS One 8: e67227.

39. Chiodi I, Mondello C (2012) Telomere-independent functions of telomerase in nuclei, cytoplasm, and mitochondria. Front Oncol 2: 133.

40. Burke LS, Hyland PL, Pfeiffer RM, Prescott J, Wheeler W, et al. (2013) Telomere length and the risk of cutaneous malignant melanoma in melanomaprone families with and without CDKN2A mutations. PLoS One 8: e71121.

This article was originally published in a special issue, Skin Cancer handled by Editor(s). Dr. Homer S. Black, Baylor College of Medicine, USA 